\title{
Questions about and speculations on the incidence of idiopathic polyhydramnios by fetal gender
}

\author{
Tae-Hee $\mathrm{Kim}^{1} \cdot$ Jun-Mo $\mathrm{Kim}^{2} \cdot$ Hae-Hyeog Lee ${ }^{1}$
}

Received: 25 January 2015 / Accepted: 9 March 2015/Published online: 19 March 2015

(C) Springer-Verlag Berlin Heidelberg 2015

Sir,

I wish to comment on the report by Stanescu et al. [1] on fetal gender bias in the incidence of idiopathic polyhydramnios (IPH). The finding that polyhydramnios is more common in male fetuses is of great interest. Below, I offer several observations and suggest points requiring clarification. First, I suggest that multiple regression analysis would help to further define the relationship between IPH and fetal gender. Second, I wonder whether Stanescu et al. [1] would care to advance a hypothesis that might explain the correlation between gender and IPH? I consider that the following information is pertinent: nocturnal enuresis (NE) occurs in $20-25 \%$ of children by 4 years of age, and is more prevalent in males than females [2]. The exact pathophysiology of the condition remains unknown, but several factors may be involved. One theory is that children exhibiting high-level nocturnal polyuria may experience difficulties in antidiuretic hormone secretion because of differences in their circadian rhythms [2]. Also, limited functional bladder capacity could affect the urine secretion [2]. No pathophysiological explanation of the betweengender difference in NE incidence has been forthcoming

This comment refers to the article available at doi:10.1007/s00404014-3504-1 and an author's reply to this comment is available at doi:10.1007/s00404-015-3690-5.

Jun-Mo Kim

urojun@schmc.ac.kr

1 Departments of Obstetrics and Gynecology, Soonchunhyang University College of Medicine, Bucheon 420-767, Republic of Korea

2 Department of Urology, Soonchunhyang University Bucheon Hospital, 170 Jomaru-ro, Wonmi-gu, Bucheon-si, Gyeonggi-do 420-767, Republic of Korea
[2]. After we read the report of Stanescu et al. [1], I developed some ideas for future studies on IHP. During measurement of the amniotic fluid index (AFI), is it possible that the time taken to evaluate mild polyhydramnios varied? Stanescu et al. [1] study contains no data on nighttime AFI. However, distinguishing daytime from nighttime AFI might be important when IPH is mild. If the incidence of AFI increases at night, a hypothesized relationship between IPH and nocturnal enuresis or polyuria, would be supported. If IPH is present, it might be instructive to measure bladder capacity after delivery. Is there any relationship between IPH and bladder capacity? We recommend that IPH patients receive long-term followup; we also wonder whether such children develop NE? The Stanescu et al. [1] study raises several urological questions. We suggest that further discussion of mechanisms that could underlie IPH pathophysiology would be instructive; given the finding that IPH prevalence is higher in male fetuses.

Acknowledgments This work was supported in part by the Soonchunhyang University Research Fund.

Conflict of interest No competing financial interests exist.

\section{References}

1. Stanescu AD, Banica R, Olaru G, Ghinda E, Birdir C (2014) Idiopathic polyhydramnios and fetal gender. Arch Gynecol Obstet. doi:10.1007/s00404-014-3504-1

2. Yeung CK (2003) Nocturnal enuresis (bedwetting). Curr Opin Urol 13:337-343 